\title{
Energy Efficient Computation of Data Fusion in Wireless Sensor Networks Using Cuckoo Based Particle Approach (CBPA)
}

\author{
Manian Dhivya ${ }^{1}$, Murugesan Sundarambal' ${ }^{2}$, Loganathan Nithissh Anand ${ }^{3}$ \\ ${ }^{1}$ Department of EEE, Anna University of Technology Coimbatore, Coimbatore, India \\ ${ }^{2}$ Department of EEE, Coimbatore Institute of Technology, Coimbatore, India \\ ${ }^{3}$ Department of Mechanical Engineering, Anna University of Technology Coimbatore, Coimbatore, India \\ E-mail: \{saidhivya1, nithisshanand\}@gmail.com \\ Received February 27, 2011; revised March 26, 2011; accepted March 30, 2011
}

\begin{abstract}
Energy efficient communication is a plenary issue in Wireless Sensor Networks (WSNs). Contemporary energy efficient optimization schemes are focused on reducing power consumption in various aspects of hardware design, data processing, network protocols and operating system. In this paper, optimization of network is formulated by Cuckoo Based Particle Approach (CBPA). Nodes are deployed randomly and organized as static clusters by Cuckoo Search (CS). After the cluster heads are selected, the information is collected, aggregated and forwarded to the base station using generalized particle approach algorithm. The Generalized Particle Model Algorithm (GPMA) transforms the network energy consumption problem into dynamics and kinematics of numerous particles in a force-field. The proposed approach can significantly lengthen the network lifetime when compared to traditional methods.
\end{abstract}

Keywords: Cuckoo Search, Generalized Particle Model, Energy Efficiency, Clustering, Sensor Networks

\section{Introduction}

Wireless sensor networks (WSNs) facilitate innovative applications and necessitate non-conventional paradigms for protocol design. Sensor node is a tiny device that includes three basic components: a sensing subsystem for data acquisition from the physical surrounding environment, a processing subsystem for local data processing and storage, and a wireless communication subsystem for data transmission [1]. Their structure and characteristics depend on their electronic, mechanical and communication limitations but also on application-specific requirements [2].

Based on the architecture and power breakdown, several approaches are considered simultaneously to reduce power consumption in wireless sensor networks. The main enabling techniques at a very general level are Clustering Schemes, Routing Protocols, Duty Cycling Schemes, Data Driven approaches and Mobility [3]. Clustering, as potentially the most energy efficient organization, has witnessed wide application in the past few years [4] and numerous clustering algorithms have been pro- posed for energy saving. Hence efficient data clustering techniques must be used to reduce the data redundancy and in turn reduce overhead on communication [5]. Low Energy Adaptive Clustering Hierarchy (LEACH) and Hybrid Energy Efficient Distributed clustering (HEED) are the traditional approaches to effective data-gathering protocols in WSNs. Energy consumption in multi-cluster sensor networks is also explained [6]. A protocol proposed in [7] optimizes the network performance under the metric of information rate per Joule while ensuring a given quality of service (QoS). QoS effectively exemplify the time and energy required to collect the packets .The traditional approach of repeated clustering makes communication and processing overheads. $\mathrm{Nu}-$ merous strategies have been projected so far, incorporating optimization techniques, but the need for energy consciousness remain as a tough issue.

This paper proposes a new optimization based data collection scheme incorporating Cuckoo Search (CS) and Generalized Particle Model Algorithm (GPMA). The formulated technique of combining the above algorithms is stated as Cuckoo Based Particle Approach (CBPA). 
The aspect of formulating this technique is incorporation of constraints that helps in energy efficient data collection or fusion, by eliminating data redundancy and minimization of energy consumption. Cuckoo Search is performed to form sub-optimal data fusion chains. The collected information is transmitted to the base station via cluster heads through GPM algorithm. In the GPM algorithm energy constraints are incorporated and the information is routed in shortest path. The proposed technique shows better performance in optimum number of clusters, total energy consumption and prolonging of network lifetime.

The rest of the paper is organized as follows. The problem formulation and Energy Model is explained in Section 2. Basics of Cuckoo Search and Generalized Particle Approach Algorithm are explained in Section 3. Cuckoo Based Particle Approach (CBPA), chain formation, proposed methodology is explained in Section 4. Results and analysis are described in Section 5. Conclusions are drawn in Section 6.

\section{Problem Formulation and Energy Model}

The aim of this paper is minimization and conservation of energy of WSNs. To minimize the usage of energy sensor nodes are formed as static clusters in first phase, by Cuckoo Search. The radio model adopted is, stated by Heinzelman et al [8]. The following are the most widely used assumptions and model in sensor network simulation and analysis [9].

Nodes are dispersed randomly following a uniform distribution in a 2-dimensional space and the location of the Base Station (BS) is known to all sensors. The nodes are capable of transmitting at variable power levels depending on the distance to the receiver. The nodes are unaware of their location. The nodes can estimate the approximate distance by the received signal strength if the transmit power level is known, and the communication between nodes is not subject to multi-path fading. A network operation model similar to that of LEACH and HEED [10] is adopted here, which consists of rounds. Each round consists of a clustering phase followed by a data collection phase.

$$
E_{T x}\left\{\begin{array}{l}
l \cdot E_{\text {electrical }}+\varepsilon_{f s} \cdot d^{2} \text { for } 0 \leq d \leq d_{\text {crossover }} \\
l \cdot E_{\text {electrical }}+\varepsilon_{m p} \cdot d^{4} \text { for } d \geq d_{\text {crossover }}
\end{array}\right.
$$

The amount of energy consumed for transmission $\mathrm{E}_{\mathrm{Tx}}$, of $l$-bit message over a distance $\mathrm{d}$ is given by Equation (1).

$$
E_{R x}=l \cdot E_{\text {electrical }}
$$

where $E_{\text {electrical }}$ is the amount of energy consumed in electronic circuits, $\varepsilon_{f s}$ is the energy consumed in an ampli- fier when transmitting at a distance shorter than $d_{\text {crossover, }}$ and $\varepsilon_{m p}$ is the energy consumed in an amplifier when transmitting at a distance greater than $d_{\text {crossover }}$. The energy expended in receiving a $l$-bit message is given by the above Equation (2).

\section{Background Work for Data Fusion}

Cuckoo search (CS) is an optimization algorithm developed by Xin-She Yang and Suash Deb in 2009. It is a novel algorithm which is inspired by the obligate brood parasitism of some cuckoo species by laying their eggs in the nests of other host birds of other species. It is more efficient than Genetic Algorithm and Particle Swarm Optimization to adapt to wider class of optimization problems [11].

In Figure 1 the Pseudo code for Levy Flights is given. The quality or fitness of a solution is proportional to the value of the objective function for the optimization problem.

The CS is based on three idealized rules:

1) Each cuckoo lays one egg at a time, and dumps its egg in a randomly chosen nest

2) The best nests with high quality of eggs will carry over to the next generation

3) The number of available host's nests is fixed, and the egg laid by a cuckoo is discovered by the host bird with a probability $P_{\alpha}$. The worst nests are discovered and dumped from further calculations.

$X$ is related to the new solution, for an $i^{\text {th }}$ cuckoo and then levy is performed as given in Equations (3) and (4).

$$
\begin{gathered}
X_{i}^{(t+1)}=X_{i}^{(t)}+\alpha^{*} \operatorname{Levy}(\tilde{n}) \\
X_{i}^{(t+1)}=X_{i}^{(t)}+\alpha^{*} E_{t}
\end{gathered}
$$

\subsection{Generalized Particle Model Algorithm}

Generalized Particle Model given by Dianxun Shuai [12], finds its application in many fields of network oriented applications. It is similar to Swarm Intelligence (SI) technique. This algorithm eradicates the unknown empirical performance and much computation time. For a multi-objective optimization problem, the algorithm controls the parameters in parallel or dispersedly.

The GP model consists of numerous particles and forces, with each particle having its own dynamic equations to represent the network entities and force having its own time-varying properties to represent various social interactions among the network entities. Each particle in GP has four main characteristics [13]:

1) Each particle in GP has an autonomous self- driving force, to embody its own autonomy and the personality of network entity. 


\section{begin}

Objective function $f(x), x=(x 1, \cdots, x d)^{T}$

Generate initial population of

$n$ host nests xi $(i=1,2, \ldots, n)$

while ( $\mathrm{t}<$ MaxGeneration) or

(stop criterion)

Get a cuckoo randomly by Levy

flights

evaluate its quality/fitness Fi

Choose a nest among n (say, j)

randomly

if $(\mathbf{F i}>\mathbf{F j})$,

replace $\mathbf{j}$ by the new

solution;

end

Fractions (pa) of worse nests

are abandoned and new ones

are built;

Keep the best solutions (or nests

with quality solutions);

Rank the solutions and find the

current best

end while

Post process results and visualization

End

Figure 1. Pseudo code for cuckoo search via levy flights.

2) The dynamic state of every particle in GP is a piecewise linear function of its stimulus, to guarantee a stable equilibrium state.

3) The stimulus of a particle in GP is related to its own objective, utility and intention, and to realize the multiple objective optimizations.

4) There is variety of interactive forces among particles, including unilateral forces, to embody various social interactions in networks.

The GPM is suitable for MAS's problem-solving in these more complex environment: multi-autonomy agents, multi-type coordination, multi-objective optimization, higher-degree parallelism, and random and emergent events [14].The assignment matrix of task allocation and resource assignment in MAS,

$$
S(t)=\left[S_{i k}(t)\right]_{n \times m},
$$

where, $S_{i j}(t)=\left(a_{i j}(t), p_{i j}(t), \zeta_{i j}(t)\right)$.

\subsubsection{GP Algorithm}

In Figure 2, the algorithm for Generalized Particle Model is given. The figure illustrates the necessary steps in devising the model. A particle may be driven by numer- ous forces that are produced by the force-field, other particles and by own. The approach incorporates hybrid energy functions to maintain the advantages of the traditional approaches and to eliminate the deficiencies of the same. The gravitational force produced by the force field tries to drive a particle to move towards boundaries of the force-field, which embodies the tendency that a particle pursues maximizing the aggregate benefit of systems [15]. In Figure 3, the pushing or pulling forces produced by other particles are used to embody social coordination's among agents. The self-driving force produced by a particle itself represents autonomy and personality of individual agents. Under the exertion of resultant forces, all the particles may move concurrently in a force-field [16]. In this way, the GPM transforms the

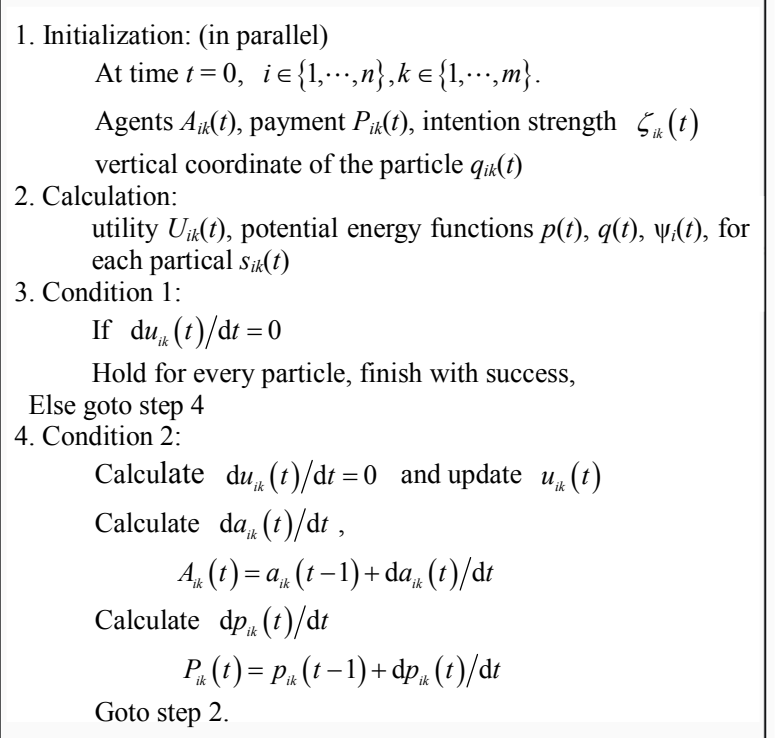

Figure 2. Algorithm for generalized particle model.

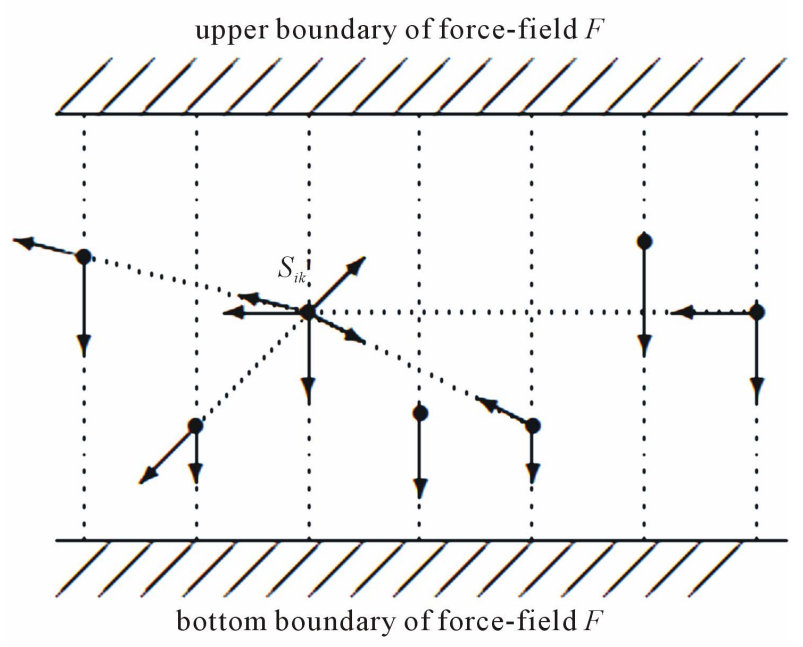

Figure 3. Generalized particle model. 
problem-solving process in Multi agent system (MAS) into kinematics and dynamics of particles in the forceield.When all the particles reach their equilibrium states, the solution to the optimization of task allocation and resource assignment is obtained.

\section{Cuckoo Based Particle Approach (CBPA)}

The energy function for the Cuckoo Search is designed as [17];

$$
f(d f i)=\sum_{i=1}^{n-1}(100 * d i)
$$

The above equation is derived from Equation (2), by considering the value of $\varepsilon_{m p}$ as $100 \mathrm{pJ} / \mathrm{bit} / \mathrm{m}^{2}$ for $\mathrm{n}=2$, i.e.; communication range between the sensors. The fitness function $F$, is considered in this problem for minimization of energy and maximization of lifetime of the nodes.

\subsection{Proposed Algorithm for Data Fusion}

Input: A set of $\mathrm{N}$ sensor nodes in randomly deployed field and a base station.

Step 1: Initialization:

Select the number of sensor nodes, cuckoo nests, eggs in nests to start the search. Initialize the location and energy of nodes and the location of base station.

Step 2: Formation of Static Clusters:

The clusters are formed, by Cuckoo Search technique. Each egg in a nest corresponds to a sensor node. A group of $\mathrm{M}$ nests are chosen with $\mathrm{N}$ eggs in it. The probability of choosing the best egg or quality egg is done by random walk. Step size and Levy angle is updated in each iteration. In turn the nests are updated. The optimal solution i.e.; best egg - high energy node is taken as cluster head in context to energy, distance between the nodes and distance to the base station.

The worse nets are abandoned in normal Cuckoo Search. In order to compensate the unequal energy dissipation, the worse nets (or) least energy nodes are allowed to join the cluster as non cluster head nodes, in the proposed approach. The less energy nodes join the proximity cluster heads to form cluster. The cluster formation is done by appropriate advertisement of cluster-head to all other nodes to join a particular cluster. The cluster head is not permanent. In each run, according to the residual energy of the nodes, the cluster head is periodically changed. This helps to eradicate the communication overhead and redundancy.

Step 3: Shortest Path Routing:

After the clusters are formed, the Cluster Heads (CHs) fuse or aggregate the information before forwarding it to the base station. The energy model incorporates free space radio model followed by all nodes. The inter cluster and intra cluster routing via shortest path is to be performed based on the application. Intra cluster refers to communication between cluster-head and non clusterhead nodes within the cluster. Inter cluster communication refers to communication between the clusters. For intra cluster communication, the most widely used methodology as followed by the basic LEACH algorithm concept is TDMA Scheduling- Time Division Multiple Access Scheduling is followed.

For inter cluster communication, Generalized Particle Model is used. The objective of using GPM approach is optimization of route and extension of the network lifetime. GPM is given in many types according to the network optimization problems by Dianxun Shuai. Normally, communication between two Wireless Sensor Nodes happens, when there is no other interfering node between two nodes. It is assumed that there exists wireless path and link between two nodes during communication.

The Generalized Particle Model transforms the network shortest path problem into kinematics and dynamics of numerous particles in a force-field. Nodes are considered as particles, and utility, overall utility, potential energy due to gravitational force, potential energy due to interactive force of particles are calculated in each iteration. It is personified in the described model, as the resultant forces on the particles are high, the particles also move fast. Then the particles are tested for stability condition. If the particles are stable, then the algorithm is terminated successfully. Else the particles are updated, with the hybrid energy equations to obtain the optimal solution.

The shortest path is calculated by the link cost of each links. The link cost is substituted as the residual energy of nodes; with context to the distance to the communicating nodes. In more brief terms the residual energy of cluster head to communicating cluster head is to be considered for communication of the sensed data. After several numbers of iterations, the optimal path to transmit the data to the base station is being established.

The important steps in Cuckoo Based Particle Approach (CBPA) are listed in Figure 4.

\section{Results and Analysis}

In this section the performance of the proposed technique is evaluated via simulation results. The network model is simulated using MATLAB. The results are summarized after running several iterations.

The focus on this paper, as by the objective function, is minimization of energy and maximization of lifetime. The simulation Parameters are listed in Table $\mathbf{1}$. 


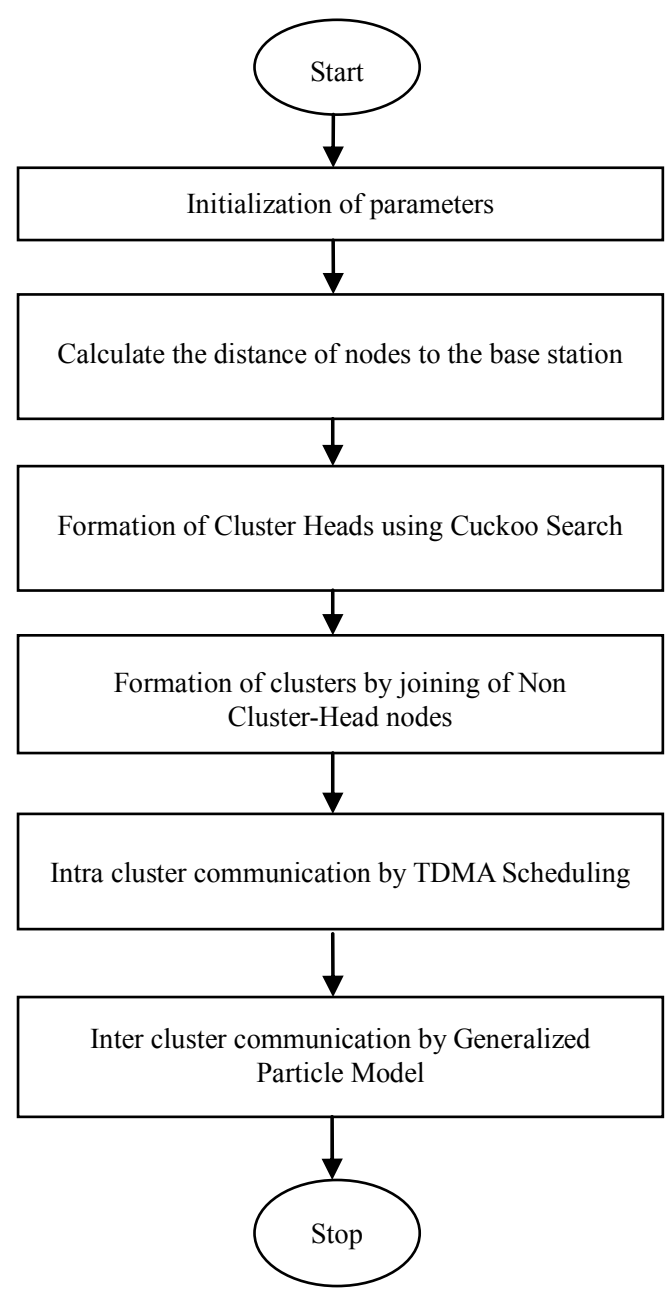

Figure 4. Flowchart of cuckoo based particle approach.

Table 1. Simulation parameters.

\begin{tabular}{ll}
\hline Sensor deployment area & $\begin{array}{l}100 \mathrm{~m} * 100 \mathrm{~m} \\
(50 \mathrm{~m}, 150 \mathrm{~m})\end{array}$ \\
\hline Base station location & $100-200$ \\
\hline Number of nodes & 100 bytes \\
Data Packet size & 25 bytes \\
Control Packet size & $2 \mathrm{~J}$ \\
Initial Energy & $50 \mathrm{~nJ} / \mathrm{bit}$ \\
$E_{\text {electrical }}$ & $10 \mathrm{pJ} / \mathrm{bit} / \mathrm{m}^{2}$ \\
$\varepsilon_{f s}$ & $0.0013 \mathrm{pJ} / \mathrm{bit} / \mathrm{m}^{4}$ \\
$\varepsilon_{m p}$ & $\mathrm{random}$ \\
Mode of Topology configuration & IEEE 802.15 .4 \\
MAC Protocol & $1 \mathrm{~second}$ \\
Duty cycle duration & 1 \\
Cuckoo step size & $60 \mathrm{~seconds}$ \\
Round duration &
\end{tabular}

In Figure 5, the clustering energy for hundred numbers of nodes is explained. It is seen the clustering energy is less than 0.05 joules for LEACH, less than 0.04 joules for HEED and less than 0.02 joules for CBPA. In Figure 6 and 7, the network lifetime of nodes is explained in context to the first node death and rounds until the last node dies is explained.

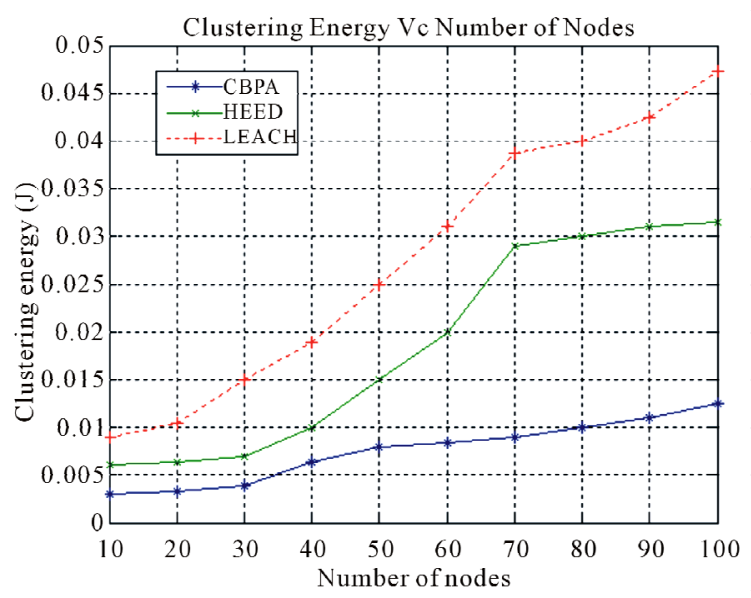

Figure 5. Clustering energy vs number of nodes.

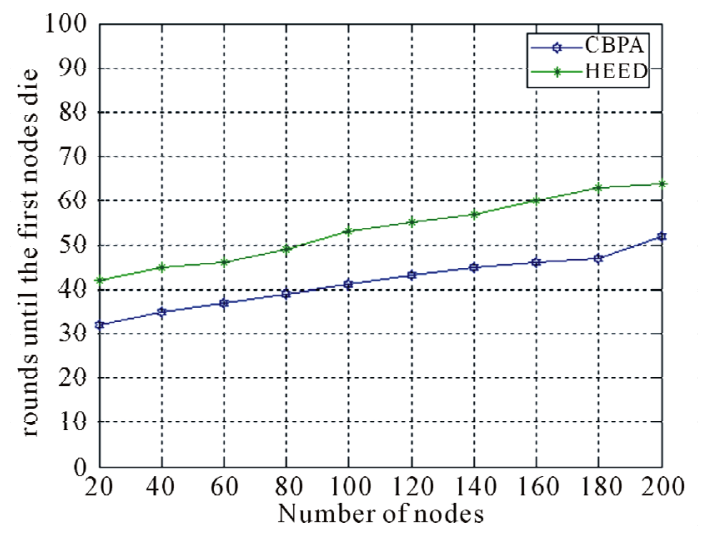

Figure 6. Rounds until the first nodes die vs. number of nodes.

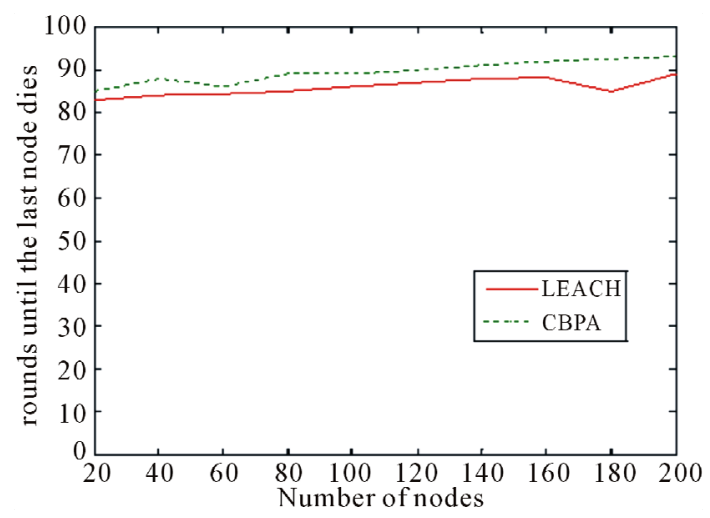

Figure 7. Rounds until the last node dies vs. number of nodes. 
In Figure 8, the average energy consumed per rounds is given. Number of alive nodes versus number of iterations is given in Figure 9. Percentage of alive nodes explains the lifetime of the network. The number of iterations is taken as 100. In Figure 10, the number of nodes versus the percentage of nodes as cluster heads is given. Normally the number of clusters is desirable to be less, as it will help in transition of node states.

The energy consumption per node increases, as the number of nodes increases. This is due to the fact that the exchange of information between neighborhood nodes and the radio channels to compete in the spectrum. The number of cluster heads is high in LEACH and HEED when compared to CBPA. As the node density increases, depending on the application there are chances for the number of clusters to increase or decrease in the traditional approaches. But in the proposed technique, it is evident that the cluster head Percentage is within the range.

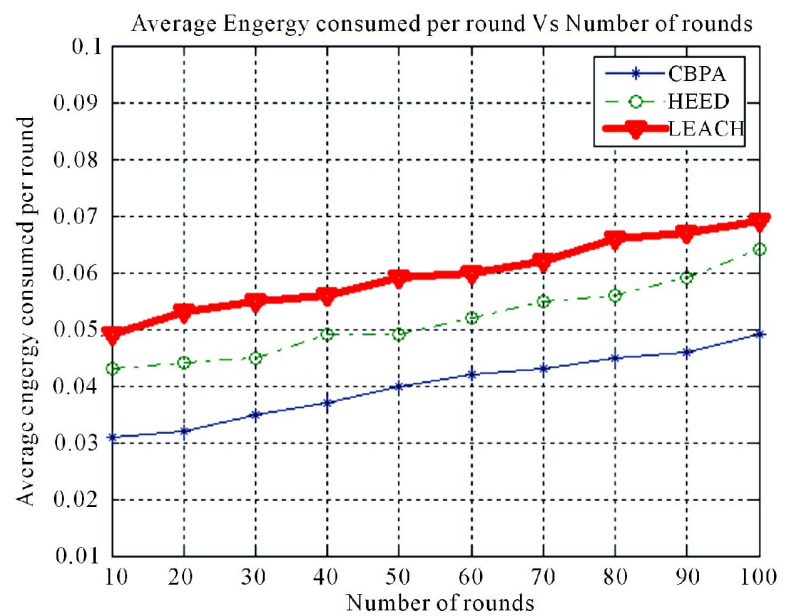

Figure 8. Average energy consumed per round vs number of rounds.

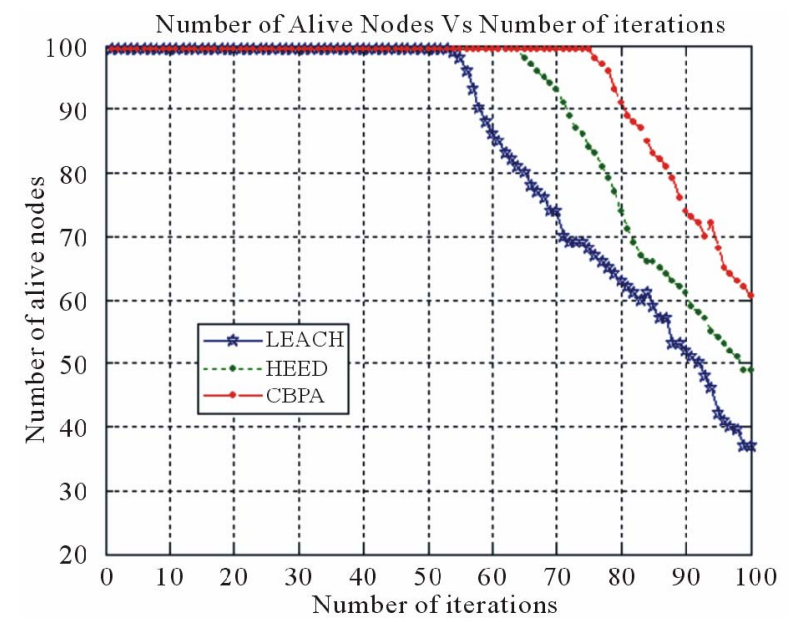

Figure 9. Number of alive nodes vs number of iterations.

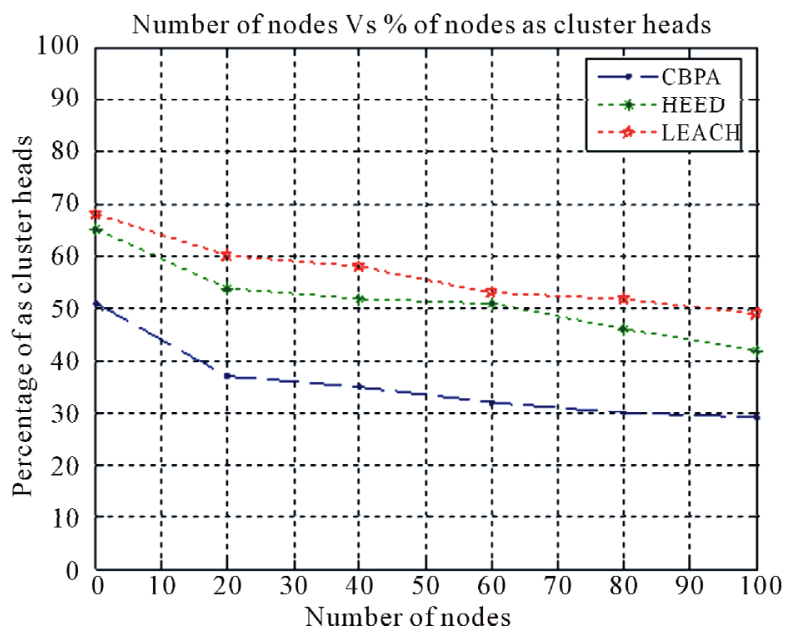

Figure 10. Number of nodes vs \% of nodes as cluster heads.

\section{Conclusions}

The cuckoo Based Particle Approach is developed to achieve energy efficient Wireless Sensor Network and multimodal objective functions. In this paper cuckoo search is applied for cluster head selection and formation of clusters among the Sensor nodes. The proposed CBPA is compared with the standard LEACH protocol and HEED protocol. The simulation results exhibits that CBPA produces comparable results mainly due to optimal search process in cluster formation and allocation of appropriate paths in transmission of sensed data. The developed suboptimal algorithm reduces complexity in chain formation and prolongs the longevity of the Sensor Network. The results are obtained by running more number of simulations. The hybrid approach offers consistency in the cluster formation, minimal number of clusters, average energy consumption and energy consumption per rounds.

In future, multi objective constraints are to be considered to obtain a realistic communication environment, with scaling and system complexity. Hybrid Optimization techniques combined with cross-layer design and Machine/Parameter learning is a challenging issue in research arena.

\section{References}

[1] F. Akyildiz, W. Su, W. Sankarasubramaniam and E. Cayirci, "A Survey on Sensor Networks," IEEE Communication Magazine, Vol. 40, No. 8, August 2002, pp. 102-114.

[2] F. P. Ferentinos, T. A. Tsilgiridis, "Adaptive Design Optimization of Wireless Sensor Networks Using Genetic Algorithms," Computer Networks, Vol. 51, No. 4, 2007, pp. 1031- 1051 .

[3] M. Dhivya, M. Sundarambal and L. N. Anand, "A Review of Energy Efficient Protocols for Wireless Sensor 
Networks," Proceedings of 1 st International Conference on Modeling, Control, Automation and Communication, Tamilnadu, 20-21 December 2010, pp. 273-278.

[4] D. Wei, "Clustering Algorithms for Sensor Networks and Mobile Ad Hoc Networks to Improve Energy Efficiency," Ph.D. Thesis, University of Cape Town, Rondebosch, September 2007.

[5] A. G. Akojwar and R. M. Patrikar, "Improving Life Time of Wireless Sensor Networks Using Neural Network Based Classification Techniques with Cooperative Routing," International Journal of Communications, Vol. 2, No. 1, 2008, pp.75-86.

[6] T. F. Shih, "Particle Swarm Optimization Algorithm for Energy-Efficient Cluster-Based Sensor Networks," IEICE Transactions on Fundamentals, Vol. E89-A, No. 7, 2006, pp. 1950- 1958.

[7] Q. Zhao and L. Tong, "Energy-Efficient Information Retrieval for Correlated Source Reconstruction in Sensor Networks," IEEE Transactions on Wireless Communications, Vol. 6, No. 1, 2007, pp. 157-165. doi:10.1109/TWC.2007.04885

[8] W. Heinzelman, A. Chandrakasan and H. Balakrishnan, "An Application-Specific Protocol Architecture for Wireless Microsensor Networks," IEEE Transactions on Wireless Communications, Vol. 1, No. 4, 2002, pp. 660-670.

[9] N. Aslam, S. Sivakumar, W. Phillips and W. Robertson, "Energy Efficient Cluster Formation Using a Multi-Criterion Optimization Technique for Wireless Sensor Networks," Proceedings of IEEE International Conference on Consumer Communications and Networking, Las Vegas, 11-13 January 2007, pp. 650-654.

[10] O. Younis and S. Fahmy, "Heed: A Hybrid, Energy- Efficient, Distributed Clustering Approach for Ad Hoc Sensor Networks," Transactions on Mobile Computing,
Vol. 3, No. 4, 2004, pp. 660-669.

[11] X.-S. Yang and S. Deb, "Cuckoo Search via Levy Flights," Proceedings of World Congress on Nature \& Biologically Inspired Computing, New Delhi, 9-11 December 2009, pp. 210-214.

[12] D. X. Shuai, X. Wang and R Gong, "A Generalized Particle Model for Social Coordination and Autonomy in MAS," Proceedings of the 2nd IEEE International Conference on Services Systems and Services Management, Chongqing, 13-15 June 2005, pp. 985-990.

[13] X. Feng, F. C. M. Lau and D. X. Shuai, "A New Generalized Particle Approach to Parallel Bandwidth Allocation," Computer Communications, Vol. 29, No. 18, 2006, pp. 3933-3945.

[14] D. X. Shuai, Q. Shuai, Y. M. Dong and L. J. Huang, "Problem-Solving in Multi-Agent Systems: A Novel Generalized Particle Model," Proceedings of the 1st IEEE International Multi-Symposiums on Computer and Computational Sciences, Hangzhou, 20-24 June 2006, pp. 322-329.

[15] D. X. Shuai and X. Feng, "Distributed Problem Solving in Multiagent Systems: A Spring Net Approach," IEEE Intelligent Systems, Vol. 20, No. 4, 2005, pp. 66-74.

[16] D. X. Shuai, B. Zhang, C. P. Lu, "A New Generalized Particle Dynamics Model for Software Cybernetics," Proceedings of IEEE International Computer Software and Applications Conference, Chicago, 17-21 September 2006, pp. 240-245.

[17] A. Chakraborty, K. Chakraborty, S. K. Mitra and M. K. Naskar, "An Energy Efficient Scheme for Data Gathering in Wireless Sensor Networks Using Particle Swarm Optimization," Journal of Applied Computer Science, Vol. 6, No. 3, 2009, pp. 9-13. 